Virtual Mentor. November 2004, Volume 6, Number 11.

doi: 10.1001/virtualmentor.2004.6.11.ccas3-0411

Clinical Cases

\title{
Who Is That? Expanding the Clinical Encounter
}

\section{Patients need to completely understand the role of any non-medical personnel present during a procedure and have the right to refuse their attendance.}

\author{
Commentary by Vijaya Arekapudi, MD
}

Emily Watson, MD, was in a hurry. Ripping off her soiled gown and balling up her used gloves, she swung by the trash on her way out of room 3C. The patient within was waiting for her epidural, but Mrs. Sanchez in 21B was ready to deliver her twins. It was 2:00 pm after a night of being the OB resident on-call, and Emily was within a few hours of heading home to get some much-needed sleep. "She signed the consent form when she got here," one of the labor and delivery nurses told Emily as they half-walked, half-ran down the hall. "For that fetal oxygen study, and the company reps are here since it's the middle of the afternoon." Emily sighed. A local biotechnology and medical equipment company was working with the Women's Hospital to test a new device for monitoring fetal oxygen levels during labor and delivery. Whenever possible, the company liked to have representatives in the delivery room to monitor the use of the equipment. Prospective participants signed a detailed consent form prior to enrolling in the study, but Emily liked to verify with the staff member obtaining consent that the patient understood the clause granting permission for nonhospital staff to observe the birth. "Who obtained consent?" she asked, knowing without hearing the answer that it was someone from the earlier shift who had since gone home.

As she entered Mrs. Sanchez's room, Emily vaguely noticed the 2 gowned individuals who followed her and the nurse inside. Familiar with protocol, they unobtrusively stayed to the side of the room as Emily adjusted a fresh gown and gloves while the nurses helped position a flushed Mrs. Sanchez into the stirrups. "Are you ready to push?" Emily asked her, following a brief internal exam. "Your cervix is completely dilated and those babies are on their way!"

Mrs. Sanchez nodded weakly, her husband standing at her shoulder. This was her first pregnancy and she had been in labor for 18 hours. The nurses moved to help hold her knees and she reached up to grip her husband's hand. "What do you think?" she asked him, smiling weakly. "One of each," he replied.

"Okay Eva," Emily said. "Remember those classes, and let's meet your twins." As Eva took a deep breath and prepared to push, she suddenly noticed the unfamiliar man and woman who had quietly moved to the foot of her bed. On a tour of the hospital as part of her prenatal class, Eva and her husband had been told about the members of the medical team who would be present for the birth of her children. After a night of being in labor, she had been introduced to all of the nurses, medical students, and residents multiple times. "Wait a minute," she said, panicked. "Who are they? What are they doing here?" she asked Emily.

\section{Commentary}

Mrs. Sanchez's confusion as to the presence of 2 strangers in her room is the end-point of several mistakes that have been made while dealing with her labor and delivery. While a hectic maternity ward and a tired resident are not unusual, these factors do not obviate the errors that occurred here.

Before taking over responsibility for the patient care in labor and delivery from the previous resident, Dr. Watson 
should have been given all of the maternity patients' information, including Mrs. Sanchez's. With this information about what is occurring in labor and delivery, Dr. Watson would then know who her patients are and what their conditions and labor statuses are, as well as any special considerations, such as the participation in the current study.

Since Dr. Watson is a resident, she needs to be supervised by an OB attending physician. Considering that Mrs. Sanchez is delivering twins, a high-risk delivery that might need to be done in a C-section room (depending on the presentation of the babies), Dr. Watson's lack of supervision by an experienced obstetrician is surprising. Unless Dr. Watson is a chief resident with a great deal of experience, she needs to be supervised. This lack of oversight is not directly related to Mrs. Sanchez's confusion, but the presence of a supervisor might well have ameliorated, if not completely eased, the situation.

These errors illustrate some fundamental systemic problems that go hand-in-hand with Mrs. Sanchez's confusion. The previous resident (assuming he or she was the one who obtained consent) should have clearly explained who would be attending Mrs. Sanchez's delivery and, if possible, should have introduced her to the company representatives prior to her delivery. Communication between residents would have at least allowed Dr. Watson to know who had, in fact, obtained consent.

Obtaining consent in this situation would require explaining to Mrs. Sanchez what the company representatives would be doing in her room as well as explaining what a fetal oxygen monitor is. Given the description of the study, it does not appear that there would be any great risk to Mrs. Sanchez or her babies, except the partial loss of privacy inherent in allowing non-medical personnel into the delivery room. Judging by her reaction, however, this level of invasion is unacceptable to the patient. While the informed consent document might be very detailed, these salient points should have been brought to Mrs. Sanchez's attention so that her decision was truly informed and educated.

Signing an informed consent document does not necessarily imply that a patient fully understands or is comfortable with what is going to occur. Given the importance of verbal explanation in obtaining consent, a physician should always be sure that the patient can understand him or her. This includes giving clinical explanations at a level the patient can understand, as well as using translators to make sure that a patient with limited English fluency fully understands what he or she is signing. Even if this was how the informed consent was obtained, it is quite possible that Mrs. Sanchez does not remember what she agreed to. If this is the case, a simple reminder might be all that is needed to make her comfortable.

Given Dr. Watson's situation, namely as a resident performing a high-risk procedure without adequate supervision, she has 2 options. If Dr. Watson feels that there is enough time to adequately explain to Mrs. Sanchez who these representatives are, then she may do so. Additionally, if after this explanation by Dr. Watson Mrs. Sanchez does not want the representatives present during delivery, even if she had previously signed a consent form and has now changed her mind, Dr. Watson should tell the representatives to leave the room. If Dr. Watson feels that there is limited time, the representatives should be asked to leave, while giving Mrs. Sanchez a brief explanation as to their presence so she is not left confused. The most important aspect of Dr. Watson's decision-making process is to give priority to Mrs. Sanchez and her babies before all others.

Vijaya Arekapudi, MD, studied obstetrics and gynecology at the College of Physicians and Surgeons of Bombay and then completed her post-graduate training at Illinois Masonic Medical Center in Chicago, IL. Dr. Arekapudi is an elected fellow of the American College of Gynecology and Obstetricians and the Chicago Gynecological Society.

The people and events in this case are fictional. Resemblance to real events or to names of people, living or dead, is entirely coincidental. The viewpoints expressed on this site are those of the authors and do not necessarily reflect the views and policies of the AMA.

(C) 2004 American Medical Association. All Rights Reserved. 